To summarise the treatment of cases in which no immediate indication as to the appropriate dose is afforded by clinical signs, such cases being generally tuberculous, it may be stated thus. In generalised tubercle, as phthisis and tuberculous peritonitis, larger doses than 1-30000th milligramme are to be given with the greatest caution, and in advanced cases such a dose even may be excessive. In strictly localised infections in adults the doses may range from 1-15000th to 1-4000th milligramme. In children with similar infections the doses may range from 1-25000th to 1-6000th, according to age and physique.

The indications as to dosage have designedly been slightly more dogmatic in the class of cases in which clinical evidence as to the appropriateness or otherwise of dosage is scanty or non-existent. Any hurry to increase the dose is deprecated, it being better to give several possibly nonmaximal doses than one large enough to produce a severe negative phase with a consequent set-back and the undoing of the good that may have pertained to many previous doses. The generalisation of the infection as opposed to strict localisation is the especial point to bear in mind. In the former small doses are to be insisted upon, for reasons given above.

In the preventive inoculation of typhoid fever previous experience can alone be relied on. Happily, this experience is extensive and the doses can be indicated with exactitude. 1000 million typhoid bacilli are administered and the patient is advised to rest, not necessarily in bed, for the rest of the day. A slight and varying amount of malaise, headache, and possibly joint pains will occur, but they usually pass off by the next day. Not less than ten days subsequent to the initial injection a dose of 2000 millions is given, and a similar sequence of symptoms may follow but is usually less marked than after the primary inoculation.

It is to be borne in mind that a high resistance in the circulating blood may not necessarily react in the direction of a cure. Unless the protective substances have free access to the area of infection the infective microbes have a highly beneficial nidus in which to multiply. Thus, the walls of a long-standing sinus may have a very poor bloodsupply by reason of pent-up discharge, due to obstruction to outflow or coagulation. In such a case the sinus may be flushed out with a hypertonic salt and citrate solution consisting of 4 per cent. of sodium chloride and 1 per cent. of sodium citrate in sterilised water. The hypertonicity of the solution promotes increased osmosis through the walls of the sinus and a more copious flow of lymph results; moreover, the citrate prevents coagulation and the increased flow of lymph through the sinus walls finds its way to the surface uncoagulated. The contents of a cavity, such as the peritoneal fluid in tuberculous peritonitis, the exudate in tuberculous pleurisy, or the contents of an abscess may be low in protective substances while the circulating blood contains an excess. Drainage of such a cavity is followed by the introduction of fluids rich in protective substances derived from the biood and the cavity, formerly a suitable breeding place for the infecting microbe, now becomes a highly unsuitable breeding place by reason of the increase in protective substances.

To meet the conditions as to dosage indicated in the foregoing remarks we prepare at St. Mary's various concentrations of vaccine. The different concentrations are so arranged that by quite simple calculations doses from the lowest to the highest figure indicated above may be given. The following is a list of vaccines prepared and issued :-

Tubercie raccine (I:R.). - Prepared from tubercle bacillary emulsion ; in two strengths-viz., 1-2000th and 1-5000th milligramme of dried comminuted tubercle bacilli per cubic centimetre.

Staphylocnecus vaccine.-Prepared from recently isolated virulent cultures of staphylococcus aureus and staphylococcus citreus; in three concentrations-viz., 100 millions, 200 millions, and 500 millions of cocci per cubic centimetre.

Streptococcus vaccine.-Prepared from recently isolated cultures of streptococcus obtained from cases of erysipelas; in three concentrations-viz., 5 millions, 10 millions, and 50 millions per cubic centimetre.

Gonococcus vacine. - Prepared from cultures of proved immunising power; in two concentrations-viz., 5 millions and 10 millions of cocci per cubic centimetre.

Typhoid vacoine.-Prepared from a culture that has been frequently tested as to its immunising power; in two concentrations-viz., 1000 millions and 2000 millions of bacilli per cubic centimetre.

There is a probability that a vaccine of micrococcus neoformans will shortly be issued, as evidence is accruing of the beneficial results as regards lessening of pain and retardation of growth and secondary deposits in some kinds of cancer. It will probably be issued in one concentrationviz., 50 millions per cubic centimetre. 15 millions may be regarded as an appropriate initial dose and 25 millions as an average dose.

The T.R. is prepared from Koch's bacillary emulsion obtained from Meister Lucius and Brüning. It is to be noted that the dilutions are made and standardised on the assumption that one cubic centimetre of the product as issued by Meister Lucius and Brüning contains two, and not ten, milligrammes of dried comminuted tubercle bacilli. The stock staphylococcal vaccine, as issued to the profession, is used at St. Mary's for practically all staphylococcal lesions. Very rarely is it necessary to make a speclal autogenous vaccine. The stock streptococcal vaccine is used initially in acute cases. As soon as the infecting streptococcus is isolated a special vaccine is almost invariably prepared, these being far more specific. Rarely is it possible to prepare special gonococcal vaccines, so the stock vaccine has usually to be relied on.

Practically all other "lesions, more especially those due to bacillus coli communis, are treated with specially prepared autogenous vaccines, and with regard to the latter there does not appear to be the slightest likelihood of a stock vaccine proving of any use in more than a small percentage of infections due to the colon bacillus.

\section{VACCINES IN GENERAL PRACTICE.}

\author{
BY LACHLAN GRANT, M.D., O.M. EdIN.; \\ T. H. CAMPBELL, M.B., OH.B. GLASG.; \\ AND
}

W. D. ANDERson, M.A., M.B. Cantab., M.R.C.S. EnG.

THE following cases having resisted the older forms of treatment it was decided to try the effect of vaccine therapy, and in these notes we will give a brief history of the cases, the modus operandi employed, and the results obtained.

CASE 1.-A man, aged 35 years, with a tuberculous history, consulted us in September, 1907, regarding a large swelling of two months' duration, on the left side of the neck, which extended from the angle of the jaw almost to the manubrium sterni. The case was one of advanced tuberculosis of the cervical glands which were evidently in a state of caseation and liquefaction. A few days later an incision was made into the dependent part of the swelling and the fluid contents were evacuated. The swelling subsided considerably and during the following three weeks the disease seemed to be held in abeyance. The improvement, however, was only temporary and the swelling advancing downwards another incision became necessary. In the course of another month, owing to the disease having spread over the manubrium, it was found expedient to make a third incision. The wounds were throughout treated antiseptically and all precautions were taken against the possible occurrence of mixed infection. Absolute rest in bed was enjoined, the patient's diet was regulated, and the best hygienic measures possible were adopted. This proved of little avail. The wounds remained open, sinuses discharging tuberculous débris. Indeed, an extension along the deep fascia into the mediastinum was feared. The affected part, after remaining unchanged for about three months, was subjected to the $x$ rays. Irradiation was given on three separate occasions at intervals of one week, the dose on each occasion being 15 minutes of a soft tube with 0.35 milliampères passing and the anticathode 12 inches from the diseased tissue. After each irradiation a thin friable cicatrix formed over the wounds, but it in variably broke down again after a few days. The patient had been under our care for seven months and we now decided on a vaccine treatment.

On May 11th, 1908, an estimation of the patient's opsonic index for tubercle bacillus was made, and the index was found to be $0 \cdot 7$. On the same day $1-5000$ cubic centimetre of T.R. was injected into the infrascapular region. This 
was followed on the 12 th by a slight inflammatory reaction in the neck, and the temperature, which had previously been normal, rose a degree, otherwise there was no constitutional disturbance. On the 13th the inflammatory signs passed off and the temperature fell to normal. On the 15th the wounds were covered by a thin delicate skin. On the 17th this skin was shed and the wounds were once more open. On the re 20th another injection of $1-5000$ cubic centimetre of T.R. was given, the opsonic index being again 0.7. This injection caused, 12 hours làter, inflammation in the wounds with distinct pain, which lasted for 24 hours. In four days the inflammation had subsided.

On June 1st a bluish-white line of epithelium began to appear round the wounds. On the 3rd $1-5000$ cubic centimetre of T.R. was again injected and the same clinical phenomena followed as on the previous occasions. On the 10th the condition was as follows: The upper wound was closed by thin, young, healthy cicatrix; the second wound was partly closed and showing healthy margins; and the third or lowermost wound was still open but was surrounded by healthy edge and floored with pink healthy granulation tissue. On the 15th 1-2500 centimetre of T. R. was injected, the opsonic index being at this date 0.89 (practically normal). On the 17th the two lower wounds were almost closed and three days later all were completely covered and the patient was now allowed to go out in warm weather. On the 25th 1-2500 cubic centimetre of T.R. was injected, the patient remaining in bed on the two following days. This procedure was followed after each injection till the termination of the treatment.

On July 2nd 1-2500 cubic centimetre of T.R. was injected, the opsonic index being now 0.98 . The patient was feeling in splendid health. The wounds had remained quite closed and the cicatrices were beginning to contract. On the 13th 1-5000 cubic centimetre of T.R. was injected. The scars were white and anæmic in places. On the 24th $1-5000$ cubic centimetre of T.R. was injected. The scars looked strong, tough, and white. An area about one-eighth of an inch in diameter in the centre of the lowermost scar had broken down. On the 29th this breach was diminished to half its previous size.

On August 19th 1-25000 cubic centimetre of T.R. was injected. On this date only an area of the size of a pin's head remained bare. On the 25 th the wounds were entirely closed and the cicatrices appeared fine, white, and strong. There was wonderfully little marking. The patient has remained well ever since and started work on Sept. 3rd.

CASE 2.-A man, aged 26 years, came on May 20th, 1908, complaining of an inflamed chin of a week's duration which proved to be a case of folliculitis barbæ. The infection involved the whole of the hairy part of the face and extended down the front of the neck. The chin was greatly swollen with subcutaneous abscesses and was the seat of much tension and pain. In a smear taken from one of the pustules the dominant micro-organism seemed to be the staphylococcus pyogenes aureus. There were also present a few streptococci and other pyogenic bacteria. The hair-roots were examined, but no trichophyton spores could be detected. The diseased parts were treated by epilation and by the application of boric acid and starch poultices, followed by antiseptic lotions and ointments for six weeks, but there was little or no improvement. Some of the pus was now plated, incubated, the right colony selected, and by a succession of sub-cultures a pure emulsion of staphylococei was obtained. This we attempted to sterilise by expnsing it for one hour to a temperature of $68^{\circ} \mathrm{C}$, or $2^{\circ}$ above that which is sometimes recommended, but a lonpful incubated for 12 hours on agar showed growth. We therefore decided upon sterilising the emulsion at $100^{\circ} \mathrm{C}$. for 40 minutes, the measured doses being again steamed for 20 minutes in the ampoules immediately before sealing. We argued that even if one loopful or more should prove sterile on incubation this was no proper guarantee that the rest of the emulsion was free from living bacteria, whereas one hour at $100^{\circ} \mathrm{C}$. practically eliminated all risk. Some consider that this temperature materially alters the toxins, so making the vaccine less efficient, but the favourable results obtainer in this case, with a possibly modified toxin, would make us hesitate to take any risks with bacteria which had only been subjected to the lower temperature.

On'July 4th a dose of vaccine containing $45,000,000$ staphylococci was injected. The opsonic index for this bacterium was then $1 \cdot 10$. Wright has recommended $500,000,000$ as a suitable dose, but other observers have obtained good results from smaller numbers. The patient was kept in bed for two days after the injection and this course was followed after each subsequent inoculation. All local antiseptic treatment was stopped. On the 5th there was a severe inflammatory reaction, with pain, swelling, and redness at the site of the disease. On the 6th the reaction passed off and a day later some of the pustules began to disappear. No new pustules made their appearance on the chin, but a crop came out on the right flank. On the 8th $90,000,000$ staphylococci were injected. Local reaction followed on the 9 th and did not subside until the 11th. Succeeding this there was a period of improvement, many pustules disappearing, and a crop again occurred on the right flank, disappear. ing in the course of four days. On the 15th the opsonic index was 0.9 and an injection of $125,000,000$ was followed by a sharp reaction and the appearance of a few pustules on the flank. On the 17th the reaction passed off, the pustules on the flank disappeared, and a period of improvement set in which continued steadily until the 24th, when the whole of the inflammatory process had subsided except at the point of the chin, where an area of about one square inch still exuded pus. On the 24th 200,000,000 staphylococci were injected and after the local reaction had passed off there was further improvement. All pain and tension had completely disappeared. On the 27 th the process had become limited to two small pustules on the chin and a few scabs.

On August 5th $300,000,000$ staphylococci were injected. No pustules were present on the chin on the 10th and the hair was growing luxuriantly. For the first time during the course of treatment no pustules on the flank followed this fourth and last injection. The patient has remained quite well up to the present time.

CASE 3.-A boy, aged ten years, was brought to us in the beginning of June, 1907, complaining of pain, stiffness, and swelling over the first metatarsal bone and proximal phalanx of the great toe of four months' duration. The case was one of tuberculous disease of the metatarso-phalangeal joint. Calmette's ophthalmic reaction was positive. The boy was put to bed, the foot was immobilised in a splint, and cod. liver oil and a nutritious diet were prescribed. The general condition was asthenic. In the course of a month the swelling had increased and showed signs of liquefaction. The question of an excision was considered, but it was decided to incise and evacuate. This was done at the beginning of August. Shortly after this the skin broke at two other place, and there were now three sinuses discharging. Treatment by Bier's method was initiated, the congestion being kept up for two hours twice daily. This had a distinctly salutary effect. The swelling began gradually to subside and the discharge lessened. One sinus closed completely, but the other two still remained open, discharging a little serum every day. During February, 1908, a second sinus closed, but opened again in a few days. This was repeated on two or three occasions during the next three months; the third wound never closed. The boy's general health had improved.

On June 13th, 1908, 1-10000 cubic centimetre of T.R. was injected and the patient was kept in bed the following day. There were a well-marked local reaction with congestion and increased discharge from the sinuses. On the 15th the reaction had subsided and during the following seven days there were a continued diminution of the swelling and a lessening of the discharge. On July 2nd another 1-10000 cubic centimetre of T.R. was injected. After the reaction had passed off further improvement occurred. One of the sinuses closed, while the other remained open, discharging a little serum every second or third day. At the beginning of August the remaining sinus closed, but opened up again about the middle of the month. On the 19th 1-10000 cubic centimetre of T.R. was injected. After the reaction had passed off the remaining sinus closed and remained so. 0n Sept. 1st the scars were white, soft, pliable, and depressed. Comparatively little thickening was present at the site of the lesion. The joint remained stiff. On this day an injection of 1-10000 cubic centimetre of T.R. was followed by a very slight reaction. The last closed sinus opened on the 2nd and emitted a drop or two of blood-stained serum. On the 3rd the dressing was absolutely dry, no discharge being present nor has there been any reappearance of the discharge since that date. 
CASE 4.-A man, aged 70 years, complained in December, 1907, of cough and expectoration, loss of appetite, weakness, and shortness of breath, all of one month's duration. He volunteered the information that he sweated at night. There was a moderate nocturnal elevation of temperature. On percussion the note over the apex of the right lung was tympanitic. On auscultation there were clicking râles and amphoric breathing at the same spot. There was also a distinct history of tuberculous disease at this apex two years previously. At that time tubercle bacilli were found in the sputum. The treatment adopted was on the recognised hygienic, dietetic, and therapeutic lines, and under this régime the patient's condition slightly improved.

At the end of April, 1908, the sputum was examined and was found to be teeming with encapsulated pneumococci mixed with a considerable number of Friedländer's pneumobacilli, but repeated examination by the more elaborate methods failed to detect any bacillus of tubercle. It was therefore concluded that the focus of tubercle was locked up and that we had to deal with an invasion of the right apex by pneumococci, and it seemed a rational treatment to attack these organisms with a suitable vaccine. This was therefore prepared from the patient's own strain of pneumococci. We considered that the clearing up of the pneumococcic infection would probably be a good step towards the resolution of any tuberculous process that might be going forward.

On May 29th the patient received the first dose of vaccine. This dose contained $15,000,000$ of mixed pneumococci and pneumo-bacilli, the former being largely in excess of the latter. Next day there was a very slight reaction which was followed by no discernible improvement in the patient's condition.

On June 6 th $15,000,000$ were again injected, the patient's opsonic index being then 1.6 for pneumococci. There was a slight reaction, a feeling of malaise and increased cough and expectoration, followed in the course of the day by a sense of well-being. The temperature now remained normal and the night-sweats were less troublesome. On the 10th he was allowed out of bed for an hour. On the 17th $30,000,000$ were injected. After the reaction had passed off there was further improvement. Cough and expectoration were diminished, the patient felt stronger, and was able to sit up for four hours. The appetite was much better. An examination of the sputum showed a remarkable diminution in the pneumococci and no tubercle bacilli were to be found. An opsonic estimation made on this day showed an index of $1 \cdot 0$ for pneumococci and $1 \cdot 3$ for pneumo-bacilli. On the 30 th he was allowed out on warm days.

On July 2 nd another injection of $30,000,000$ was given. A few days after this the patient was able to go for an hour's walk. The improvement was progressive and on the 27th he was able to do a little manual work. All the signs of a cavity were, however, still present at the right apex. On the 28th 10,000,000 were injected, and after the reaction had passed off the patient's condition continued to improve. He was soon well enough to work. Since early in August he has been engaged in a regular, though light, occupation, and savs that he feels better than he has done for nearly a year. There are still cough and expectoration, but the cough is slight and the expectoration very scanty. A few râles are to be heard at the right apex, but on the whole the improvement is very great indeed.

Conclusions. - The results in the foregoing cases have so far been most satisfactory and we venture to attribute this to the action of the vaccines. In those cases treated with tuberculin the following points are of note:-

1. The scars resulting were soft, pliable, and the disfigurement slight.

2. Recovery under the treatment seemed rapid, and thi implied saving of suffering and expense to the patient and anxiety to the medical attendants.

3. The absence of severe surgical measures minimised the risk of a general tuberculous infection and obviated that slight risk which always attends general anæsthesia.

4. Although opsonic estimations were only made occasionally during the treatment, they suggested that the index was being steadily raised, and from this it is probable that for some time, at least, the patients will be less susceptible to attacks by the same bacterium. Surgical measures alone may achieve this indirectly, inasmuch as they rid the body of the focus of disease, but it is questionable if they are capable of raising the standard of defence to the same high level as that induced by vaccine therapy.

It may perhaps be right to conclude by saying that much expense and valuable time were saved to the patients and to ourselves by the fact that the microscopical work, the electrical treatment, the preparation of vaccines, and the opsonic estimations were all çonducted in our own laboratories.

Ballachulish, Argyllshire.

\section{THE SANITATION OF MINES. ${ }^{1}$}

\section{By FRANK SHUFFlebothaM, M.A., M.B., B.C. Cantab.}

THE question of the sanitation of mines has been brought prominently to our notice of late years through the outbreak of ankylostomiasis in Westphalia, Belgium, and other European countries, as well as among the tin miners of Cornwall. In each district the disease has affected large numbers of workers, in some cases to such an extent as to be a great menace to the industry. The absence of sanitary arrangements underground has been the principal cause of so many men being struck down by this disease, and it has been due mainly to the institution of elaborate sanitary arrangements that the disease has been eradicated, and even then in each epidemic it has been a matter of many months, and in some districts of several years, before the disease disappeared.

Proper sanitary arrangements are desirable in mines on general hygienic grounds, but they are necessary on account of the dangers which may arise through their absence. In the first place I wish to show how ankylostomiasis can be spread among miners through the want of proper sanitation underground, and I will describe briefly the nature of the disease, so that this will be all the more easily understood.

\section{Nature of Ankylostomiasis.}

Ankylostomiasis is a disease caused by the presence of a small worm known as the ankylostoma duodenale in the upper part of the small intestine. The worm attaches itself to the mucous membrane of the bowel by means of hooks which are situated round its mouth. It does not reproduce itself within the intestine but the female lays an enormous number of microscopically small oval eggs which pass away in the fæces. The eggs hatch out into larvæ under favourable conditions of temperature and moisture and in the presence of oxygen and absence of daylight. The eggs will not hatch out in the intestine owing to the lack of free oxygen. The larvæ continue to grow and after a short time they develop a capsule. It is in this state that they enter the human body. Miners often relieve themselves underground and if they are infected with ankylostoma then the motions would be a source of danger. The hands of their fellow-workers might become contaminated with infected fæcal matter and when they take their food at "snapping-time" they would swallow some of the encapsuled larvæ which in the intestine develop into full-grown worms. The worm may live in the human intestine for several years. The disease is endemic in many tropical and subtropical countries but when found in temperate climates it is practically confined to miners and workers in tunnels.

Symptoms and diagnosis of the disease.-The symptoms are : (1) anæmia with its usual accompaniments, pallor, shortness of breath on exertion, palpitation, giddiness, and fainting ; (2) gastro-intestinal disturbances; and (3) in the outbreak in Cornwall skin symptoms were found. The posilive diagnosis can only be made when the eggs are found in the stools. For the methods of detecting the eggs I would refer to the Home Office Memorandum of Feb. 16th, 1904, and to papers by Dr. A. E. Boycott and Dr. J. S. Haldane in the Journal of Hygiene, January, 1903, and January, 1904.

It must be remembered that only a certain percentage of those infected with ankylostoma show symptoms of the disease. This is of great importance from the fact that they are just as great a menace as those whose symptoms are marked. The examination of the blood, however, will show

1 A paper read before the Industrial Hygiene Section of the Roya Institute of Public Health at the Buxton Congress on July 21st, 1908 . 